\title{
On the perceived unitization of repetitive auditory patterns
}

\author{
FRED L. ROYER \\ Cleveland VA Medical Center, Brecksville, Ohio \\ and \\ DONALD A. ROBIN \\ University of lowa, Iowa City, lowa
}

\begin{abstract}
When a cyclic pattern of sound bursts occurs, a rhythmic pattern will be heard at certain rates of presentation. When rate is increased sufficiently, the pattern is heard as a repeating unit-a repeating packet of sound bursts consisting of the whole cycle. The temporal parameters of the process of unitization that produces the perceptual phenomenon were examined. Critical burst duration for fixed interburst intervals and critical interval for fixed burst durations were obtained. An inverse linear relationship between duration and interval was found. The sum of duration and interval yielded a constant that increased as the number of elements in the pattern increased. The results are interpreted as supporting a lowpass filter model of temporal pattern organization in the auditory system.
\end{abstract}

If one listens to a sound burst that reoccurs regularly at particular intervals, a number of perceptions are possible. What one perceives depends, among other things, on the temporal structure of a given pattern and the interval over which the pattern occurs. At very, very long intervals, particularly if there are distractions present, one perceives only intermittent sound burst without pattern. As the interval is shortened, one perceives eventually a rhythmic pattern that has a perceptual beginning and end despite the cyclic structure of the temporal sequence of pattern elements. The individual elements are heard as being clearly discrete, yet they are organized into a pattern. As the interval is shortened further, which is to say that as rate is increased further, one hears a change in organization that we describe as unitization. One hears a repeating packet of sound bursts, which form a unit consisting of the whole cycle, while also hearing individual elements. When unitization occurs, the temporal structure of elements of the rhythmic pattern, particularly if it is complex, may not be identifiable. As the rate is increased further, one perceives still another change; one hears only an auditory texture, a continuous sound with a particular timbre determined by both the element structure and the tone-burst quality.

In this report, we explore the temporal parameters of

\footnotetext{
This research was supported by the Veterans Administration Medical Research Service. The authors acknowledge the valuable contributions to the study made by J. J. Gruhn, who did the computer programming and served as a subject, by D. Johnson, who served as a subject, and by $M$. Rogers, who provided technical assistance. Requests for reprints may be addressed to Fred L. Royer, 545 Spring Pond Rd, Northfield, $\mathrm{OH} 44067$.
}

the perception of unitization because the existence of this perceptual phenomenon is indicative of a processing system that behaves somewhat like a lowpass filter.

\section{Auditory Temporal Pattern Perception}

Royer and Garner $(1966,1970)$ investigated the perceptual organization of repeating (cyclic) eight- or nineelement sequences of two different sound bursts. Listeners used only a few of the eight or nine alternative elements of the sequence as the start of the pattern they perceived. The choices were lawful and appeared to be determined by the holistic structure of the pattern. The difficulty of reproducing the sequence was related to the empirically determined number of alternative organizations used by subjects.

Preusser, Garner, and Gottwald (1970), investigating the holistic organizing principles of temporal balance and progression found by Royer and Garner (1970), saw these principles as a sort of interaction between the temporal patterning of each of the sound bursts. Let $X$ and $O$ represent two different sound bursts. The cyclic sequence ...XOOXXXXOXXOOXXXXOXX..., for example, would be reported by listeners as having one of two perceptual organizations, OOXXXXOXX or XXXXOXXOO. Preusser et al. (1970) proposed that the perception of the above binary pattern might be determined by organizing processes operating on two interdigitated unary temporal patterns, ...XXXX $\mathrm{XX}^{\prime} \mathrm{XX}^{\prime \prime} \mathrm{XXXX}^{\prime} \mathrm{XX}^{\prime \prime} \ldots$ and ...OO" $\mathrm{OO}^{\prime \prime} \mathrm{O}^{\prime \prime \prime} \mathrm{OO} \mathrm{O}^{\prime \prime} \mathrm{O}^{\prime \prime \prime \prime} \ldots$, where $\mathrm{X}$ and $\mathrm{O}$ represent different sounds and the prime (') represents a silent interval. They identified two principles that operate in the perception of these half-patterns: run and gap. In general, the perceived beginning of a continuously repeating pat- 
tern is the element that starts the longest run (a sequence of Xs or Os), or the first element after the longest gap (a period of silence, or, so to speak, a run of no-tones).

Preusser et al. (1970) found that the frequency with which one of the several elements of a unary pattern was indicated by the observer as the start of the pattern was determined by the interplay of the runs and gaps in the pattern. For example, both run and gap principles predict that the perceived start of $\mathrm{XXXX}^{\prime} \mathrm{X}^{\prime \prime \prime}$ will be with the first element; thus, run and gap principles are compatible. When the principles are not compatible, gap structure tends to dominate. This is particularly so at rates between 2 and 4 elements/sec, although the run principle may retain strength at slower rates (Preusser, 1972).

The strength of the perceived pattern organization of two sound bursts (Xs and Os) in the Royer-Garner patterns (Royer \& Garner, 1966, 1970) can be explained as being determined by the interaction of these organizing principles for each of the two half-patterns. If the principles for each unary pattern (half-pattern) were compatible with one another, the organization was strong; if the principles were incompatible, the organization was weak. For example, Royer and Garner (1966) found that two alternative organizations of the cyclic pattern XXXOX$\mathrm{OXO}$ were quickly reported by listeners. The two unary patterns, $\mathrm{XXX}^{\prime} \mathrm{X}^{\prime} \mathrm{X}^{\prime}$ and ${ }^{\prime \prime} \mathrm{O}^{\prime} \mathrm{O} \mathrm{O}^{\prime} \mathrm{O}$, have constructions in which organization is predicted by only one principle, the run principle in the former and the gap principle in the latter. However, Royer and Garner (1966) found that four alternative organizations of the pattern XXOXOOXO were reported after prolonged listening. The run principle predicts organization at the first burst or element of the half-pattern $X^{\prime} X^{\prime \prime} X^{\prime}$ and the gap principle predicts organization at the fourth $X$ or seventh element. The run principle predicts organization at the second $O$ or fifth element of the half-pattern " $\mathrm{O}$ ' $O \mathrm{O}^{\prime} \mathrm{O}$ and the gap principle predicts organization at the first $O$ or third element of the sequence. Each unary pattern is predicted to be difficult to organize because the run and gap principles conflict within the pattern structure; the binary pattern is predicted to be very difficult because both principles conflict between unary pattern structures.

The run and gap principles of temporal organization place an emphasis on the relative distribution of pattern elements over some interval. The nature of the sound bursts distributed over the interval does not seem very important. In fact, the sound bursts used to generate patterns in the experiments cited above varied considerably, being buzzes, complex waveforms, and pure sinusoids. The organizational principles have been found to hold regardless of the type of burst. The durations and amplitudes of the bursts have been constant within a pattern so that whatever organization results, including a perceived accent or stress marking the pattern's beginning, does so in the absence of such other variables as duration, pauses, or intensity, which can affect the organization of two-element patterns (Handel, 1973, 1974). The evidence is consistent with our ability to hear a rhythmic pattern in music, whether the sounds are produced by brief transient sounds like a drummer's rim-shot, sustained sounds like an organist's legato passage, or any number of sound qualities produced by various instruments.

This discussion has emphasized the relative temporal packing of sounds on the perception of temporal pattern. However, there clearly are limits on the temporal organizing processes. The rate of presentation of an entire sequence of sounds affects the perception of pattern. Garner and Gottwald (1968) showed that the observation time required to describe a binary auditory sequence is a $U$ shaped function of presentation rate. Between pattern presentation rates of 0.8 and 8.0 events $/ \mathrm{sec}$, the minimum observation time was around 2.67 events/sec. Fraisse's (1982) review of related literature indicates that the ability to perceive pattern deteriorates at interevent intervals greater than 1,800 msec. The work of Garner and Gottwald (1968) indicates that the ability to analyze a pattern suffers at very slow rates, but it also indicates that the ability suffers at fast rates. The time spent listening before responding increased as pattern presentation rates increased, despite the fact that there are more cycles of the pattern in some unit time as repetition rate increases.

Apparently, analytic processes become less efficient as rate increases. Other experiments have shown similar difficulties with pattern analysis when presentation rate is greater than 10 events $/ \mathrm{sec}$. The order of sequences of various types of sounds become confused (Broadbent \& Ladefoged, 1959; Warren, Obusek, Farmer, \& Warren, 1969). The sequence becomes a unit in which elements are indistinguishable with respect to order.

The tendency to hear sequences as a unit at fast presentation rates is also found in the work of Handel and Oshinsky (1981). They found that subjects who tapped out polyrhythmic patterns gave a "unit" response when rates exceeded $.8 \mathrm{sec} /$ pattern repetition (interelement intervals of less than 200-250 msec). The polyrhythmic patterns had two trains of sound bursts occurring repetitively over some interval. For example, one train had three events occurring in some unit time and the other train had four events in that time; this pattern had a meter of "three against four." The onsets of such bursts of the two trains will coincide periodically. At fast rates, their subjects keyed their responses to this coincidence. The "unit" response does not seem to be explainable by motorresponse limitations, according to Handel and Oshinsky (1981).

As noted earlier, our many listenings to unary sequences (half-patterns of Preusser et al., 1970) indicate a perceptual phenomenon at very fast presentation rates in which a transition occurs from perception of a rhythmic pattern of separate events to a perception of a repeating packet of events. At these presentation rates, identification of a particular sequence is difficult or impossible, although two different patterns presented one after the other are clearly distinguishable from one another.

The evidence is strong that the relative distribution of sounds over time (a sequential pattern) indicated by run 
and gap lengths and the rate at which these repeat in time are critical variables in temporal organization. There is another perspective on the problem that has received considerable attention that is important in our later discussion. Martin (1972) has proposed an alternative to the run and gap principles, based on his theory that hierarchical rhythmic structure dominates auditory temporal patterns of speech and music. According to Martin, accents naturally occur in equally spaced events. An easily perceived ("good") pattern with a length of eight events is one in which accents fall on the first and third or fifth element; a difficult (" poor') pattern is one in which accents fall in other places, leading to syncopation. Sturges and Martin (1974) used 7- and 8-element binary sequences to test this theoretical position. There cannot be equal spacing of accents in a 7-element sequence, so it becomes a test of the hypothesis. In their experiment, there were either two cycles of a pattern or one cycle followed immediately by a cycle of a different pattern. Subjects were more accurate in judging whether the consecutive patterns were same or different when the sequences were 8 elements long than when they were 7 elements long; they were also more accurate in making the judgment when the sequences were "good" than when they were "poor." Since all the sound bursts used in the studies cited earlier were constant in duration and amplitude within a sequence, accent must be assumed to occur at a transition from one sound burst (say, $X$ ) to another (say, O). Martin's theory does not explain how this psychological accent is imposed or why the transition from one type of element to another will dominate perceived accent, but the work of Sturges and Martin (1974) does support Martin's general argument that there is some perceptual salience to events that are ordered so that transitions occur at an interval half that of the pattern length, at an interval a quarter of the pattern length, and so on. The incompleteness of Martin's theory is indicated by the finding that run and gap principles held for organization of the 7-element unary or halfpatterns used by Preusser et al. (1970), just as they held for 8-, 9-, and 10-element unary pattern sequences. The incompleteness is indicated, as pointed out by Jones (1981), by its inability to predict the increasing difficulty of pattern analysis as the presentation rate of the pattern increases (Garner \& Gottwald, 1968). Similarly, its incompleteness is indicated by its inability to predict a shift of pattern organization to a single unit, such as we have observed. Nevertheless, we can state the unitization phenomenon in terms of the hierarchical models of Martin (1972) and Jones (1976). It is as though increasing presentation rate moves the perceived organization to higher levels of the hierarchy until finally the apex of the hierarchical rhythmic structure is reached, at which a single unit is heard.

We now examine the implications of these experimental observations for temporal pattern perception. A model is presented that leads to predictions of the perceptual phenomenon observed at fast presentation rates.

\section{A Filter Model of Temporal Pattern Organization}

That one can perceive a rhythmic pattern while clearly hearing the elements is reminiscent of an analogous phenomenon in vision. When viewing alphanumeric characters composed of dots, one sees both the clearly defined dots and the global organization of the character. For example, if a letter, $\mathrm{G}$, is composed of dots on an imagined matrix, one sees the G-ness while simultaneously seeing dots with clearly defined edges. In Gestalt terms, there is closure of the spaces between dots that yields the Gestalt $\mathrm{G}$

Ginsburg (1978) suggested that this visual phenomenon might be modeled as the output of spatial-frequencysensitive channels. A channel sensitive to low spatial frequencies might function as a lowpass filter. The lowpass filter output would create the blurred image that would have closure and readily perceivable G-ness. The output of the two filters would be combined at some level into the percept, but the contribution of the lowpass filter output would be preserved as global organization.

We believe that this concept provides a useful step toward modeling auditory pattern organization, just as auditory analogues of visual processes developed by Bregman (1981) and Kubovy (1981) have proven useful.

First, we note that our auditory pattern signals can be analyzed by the Fourier transform into component sinusoidal frequencies having magnitude and phase. In the case of the auditory patterns we have been discussing, the spectrum may range from $0.1 \mathrm{~Hz}$ up to the limit of hearing, greater than $20 \mathrm{kHz}$, depending on the rate of the temporal pattern and the frequency of the tone or sound bursts. We are accustomed to thinking of 10 to $16 \mathrm{~Hz}$ as the lower limit of frequency analysis by the ear (e.g., Yost $\&$ Nielsen, 1977). Although that is true for pitch perception, frequency analysis of much lower frequencies (which we will call temporal frequencies) mav be accomplished by another neural mechanism. We assume, for our model, that there are at least two parallel frequency analyses performed on the input. One analysis is of pitch, the other of temporal frequency. Second, we additionally assume that the very low(temporal)-frequency analysis system has the same analytic capabilities as the pitch-frequency analysis system. Therefore, mirroring the hierarchical relations of pitch in which pitches an octave apart are perceived as being qualitatively the same except for tone height (Jones, 1976), temporal frequency relations are hierarchical so that temporal frequencies an octave apart are perceived as being the same except for "rate height." Thus, a temporal pattern retains its perceived character despite "transposition" to greater or lesser rates.

How can we model the temporal frequency analytic system? The unary pattern sequences studied by Preusser (1972) and Preusser et al. (1970) may be thought of as amplitude-modulated signals, as shown in Figure 1A. The tone or sound burst is the carrier signal, and the pulse pattern is the modulating signal. The binary pattern se- 


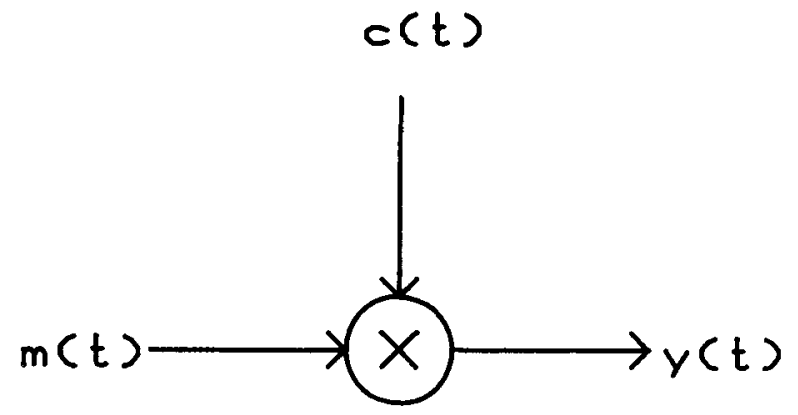

A.

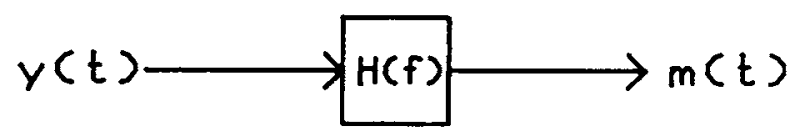

B.

Figure 1. (A) The stimulus in these experiments, $y(t)$, is an amplitude-modulated signal, produced by multiplication of a modulating signal, $m(t)$, by a carrier signal, $c(t)$. In these experiments, the carrier was a $1200-\mathrm{Hz}$ pure tone and the modulator was a pulse train. (B) In the model, the temporal pattern is the modulating signal, $m(t)$, which is recovered by passing the stimulus signal, $y(t)$, through a lowpass filter with the transfer function, $H(f)$.

quences of Royer and Garner $(1966,1970)$ may be thought of as two multiplexed amplitude-modulated signals.

In amplitude modulation of signals, the carrier signal generally has a frequency much greater than the highest modulation frequency and recovery of the modulating signal is easily accomplished by a lowpass filter, as shown in Figure 1B. An ideal filter of this type passes frequencies, up to its cutoff, without any change in amplitude, and it rejects all frequencies higher than the cutoff frequency. Ideal filters are not realized in natural systems. Typically, the filter passes frequencies up to some frequency after which the amplitudes of higher frequencies are successively attenuated.

Fourier analysis will show that a complex waveform of the type that modulates tone bursts of auditory patterns is composed of many harmonics. As the higher harmonics are successively filtered out, the definition of the edges of the pulses becomes poorer and poorer. Smoothing is produced. Depending on the cutoff frequency and other filter characteristics, the output of a lowpass filter will eliminate high frequencies and smooth the envelope of the successive bursts. As the filter cutoff frequency is lowered or, obversely, as the fundamental frequency of the cyclic pattern is increased, smoothing of the burst envelope will be greater.

According to our conception of the model, the characteristics of the filter responsible for temporal patterning will smooth the envelope of discrete sound bursts so that at some repetition rate all bursts are smoothed into a single cyclic waveform (e.g., an envelope with a node at the end of each repetition or a rectified waveform that drops to a minimum at the end of each repetition). At less than this rate, rhythmic organization can be heard. At greater than this rate, the complete modulated signal will be heard as a texture or timbre.

Viemeister (1979) proposed a lowpass filter model incorporating some nonlinearities to account for thresholds for detecting sinusoidally amplitude-modulated wideband noise. We are proposing a similar mechanism with a cutoff frequency that is much lower than the $65-\mathrm{Hz}$ cutoff of his model.

\section{Purpose}

As a first step toward developing a lowpass filter model, we examined the temporal parameters of the unitization phenomenon. For simplicity's sake, we confined our investigation to unary patterns. If unitization results from lowpass filtering, we should find some consistency in temporal parameters of stimulation necessary to produce unitization. Suppose we vary the number of bursts making up a pattern of some specified time period, say $1 \mathrm{sec}$. For some fixed time constant of a filter, there will be less smoothing of the envelope if there are three bursts spread out in time than if there are four or five in the same time period. The frequency of the three-burst pattern will have to be increased more than that of the four- or five-burst pattern to get equivalent smoothing. That is, the three bursts will have to be packed relatively more closely in time to get equivalent smoothing over the bursts.

To conclude that a lowpass filter models the phenomenon, it is necessary to establish that variation of either duration or interval will always lead to the same burstonset-to-burst-onset time. Suppose we allow the subject to adjust the duration of the burst in order to produce unitization and do so for several different interburst intervals. The observer should adjust duration so that the interval between burst onsets is constant. The longer the interval, the shorter should be the adjusted duration, and vice versa. Varying the duration of the bursts and allowing the subject to adjust the interval between bursts should produce an equivalent relationship. Expressed in terms of the frequency domain, adjusting either the duration or interval while the other is fixed will shift the fundamental of the pattern's spectrum with reference to the cutoff frequency of the filter's transfer function. It will also compress or expand the spectrum, which will have an effect on the extent of smoothing because of the extent of attenuation and rejection of higher frequency spectral components by the filter. 


\section{METHOD}

\section{Subjects}

Three subjects participated in the experiment. Two were experienced listeners (D.R. and J.J.G.), one was inexperienced (D.J.). Their ages ranged from 26 to 34 years. All subjects had normal pure-tone air-conduction audiometric thresholds.

\section{Apparatus}

Sound bursts were generated by a Wavetek Model 112 gated by a digital output from a Digital Equipment Co. PDP-11/34 computer. After amplification, the sounds were presented binaurally to the subject through Grason-Stadler TD-49 matched headphones. The sine-wave-burst output of the Wavetek always started and ended at zero degrees of phase. Computer control of sound-burst duration and interstimulus intervals was performed by machine language subroutines.

\section{Stimuli}

Sound bursts consisted of $1200-\mathrm{Hz}$ pure-tone sine waves. Their amplitude was $70 \mathrm{~dB}$ sound pressure level.

Eight different temporal patterns were presented. Let $X$ represent the tone burst of some duration (D), followed by an interval $\left(I_{1}\right)$ of silence before presentation of the next burst. Let ' represent a silent interval $\left(\mathrm{I}_{2}\right)$ equal to the sum of $D$ and $I_{1}$. The patterns presented were:
A. $X^{\prime} X^{\prime} X^{\prime \prime \prime}$
B. $X^{\prime \prime} X^{\prime} X^{\prime \prime}$
C. $\mathrm{XXX}^{\prime} \mathrm{X}^{\prime \prime \prime}$
D. $X X X^{\prime \prime} X^{\prime \prime}$
E. $X X^{\prime} X^{\prime \prime} X^{\prime}$
F. $X X X^{\prime} X^{\prime} X^{\prime}$
G. $X X^{\prime} X^{\prime} X X^{\prime}$
H. $\mathrm{XXXX}^{\prime} \mathrm{XX}^{\prime}$

Patterns A, C, D, F, and $\mathrm{H}$ are written in an organization that would be expected according to the run and gap principles of Preusser et al. (1970). These patterns are organized strongly in the form written and may be considered as perceptually unambiguous. Patterns B, E, and G, however, have a structure in which run and gap principles are in conflict. They are not strongly organized as written; they may be heard with more than one organization or even with a changed organization during listening. For example, pattern $\mathrm{B}$ is sometimes heard as $\mathrm{X}^{\prime} \mathrm{X}^{\prime \prime} \mathrm{X}^{\prime \prime}$ and sometimes as $\mathrm{X}^{\prime \prime} \mathrm{X}^{\prime} \mathrm{X}^{\prime \prime}$ at moderate rates. These may be considered as perceptually ambiguous patterns. The ambiguous patterns tend to have greater temporal dispersion of the elements than do unambiguous ones. Accordingly, one might expect that the rate at which unitization would be achieved with ambiguous patterns might be faster than the rate with unambiguous patterns. However, unambiguous patterns are more regular in structure than ambiguous ones, or, in the terminology of Martin (1972) and Sturges and Martin (1974), unambiguous are metrical and ambiguous are ametrical patterns. Regular or metrical patterns are those that have repetition of bursts at intervals of $T / 1, T / 2$, or $T / 4$, where $T$ is the total time for a sequence that could be filled by eight bursts; irregular or ametrical patterns are those that have repetition of bursts at intervals other than these. This regularity should concentrate the spectral energy in fundamental, second, fourth, and eighth harmonics in contrast with a dispersal of energy among other harmonics not in the octave relationship with the fundamental. The difference in concentration of spectral energy may affect the ease of perceiving pattern as well as the smoothing that would occur to create unitization because of lowpass filtering.

\section{Procedure}

Each subject was tested under two experimental procedures. In Procedure A, interburst interval $I_{1}$ was fixed at $10,20,30,40$, or
$50 \mathrm{msec}$. The computer picked a pattern and ISI on the basis of a random-number algorithm and played the sequence beginning with a tone-burst duration, D, of $100 \mathrm{msec}$. The listener pressed a key to reduce the duration of $\mathrm{D}$. When the subject heard the transition to unitization, he pressed another key to indicate this. If the listener was uncertain and had not yet pressed the key signaling that unitization had occurred, he could use another key to recross the threshold by increasing the duration of $\mathrm{D}$ until he was sure there was no unitization, then returning to the other key to decrease D and reestablish threshold.

In Procedure B, burst duration D was fixed at 10, 20, 30, 40, or $50 \mathrm{msec}$. Then the pattern started with the interburst interval $I_{1}$ at $100 \mathrm{msec}$. The listener pressed a key to reduce $I_{1}$. Following the procedure outlined above, he signaled when he had experienced unitization.

In both conditions, the rate of reduction of $D$ or $I_{1}$ was rapid for a practice trial. For example, for practice trials in Procedure A, the computer interrogated the subject's key at the end of each elapsed $\mathrm{I}_{2}$. The duration of $\mathrm{D}$ was then reduced by $2 \mathrm{msec}$ so long as the subject kept the "decrement" key depressed. If released, the interval remained unchanged. The subject pushed the appropriate "unitization" key when unitization was perceived. This practice procedure estimated the threshold. The experimental trials then began. The duration $D$, in Procedure $A$, or the interval $I_{1}$, in Procedure $B$, was incremented by $10 \mathrm{msec}$ plus a randomly selected integer between -2 and +2 , then decremented by $0.5 \mathrm{msec}$ if the decrement key was down during the computer's interrogation. When the subject pressed the key indicating that unitization was heard, the duration or interval in effect at that moment was recorded by the computer. This procedure continued for five trials. The average of five trials yielded the unitization threshold.

\section{RESULTS}

The critical durations and intervals at which unitization occurred for each pattern are shown in Figure 2. Two things are clear from inspecting the figure. First, there is an inverse linear relationship between duration and interval. To obtain unitization, tone-burst duration must increase as interval decreases or interval must increase as tone-burst duration decreases. In other words, there is an additive relationship between tone-burst duration and interval that produces a constant for a given number of bursts. There is no special significance to the constant; it simply indicates that, regardless of either burst or interval duration, the burst-onset-to-onset time is constant. This constant determines the pattern's fundamental frequency at unitization. This frequency is always constant for a pattern.

Second, the patterns are ordered in the same way, whether the listener was manipulating duration or interval. When tone-burst duration is fixed, three burst patterns have critical intervals shorter than four burst patterns, which, in turn, have critical intervals shorter than five burst patterns, and so on. When intervals are fixed, three burst patterns have shorter durations than four burst patterns, which, in turn, have critical durations shorter than five burst patterns, and so on. There is a direct relationship between the number of sound bursts in the pattern and the duration or interval at which all bursts of a cycle are integrated into one perceptual unit. Patterns with more bursts achieve unitization at much longer durations or intervals than do patterns with fewer bursts. 

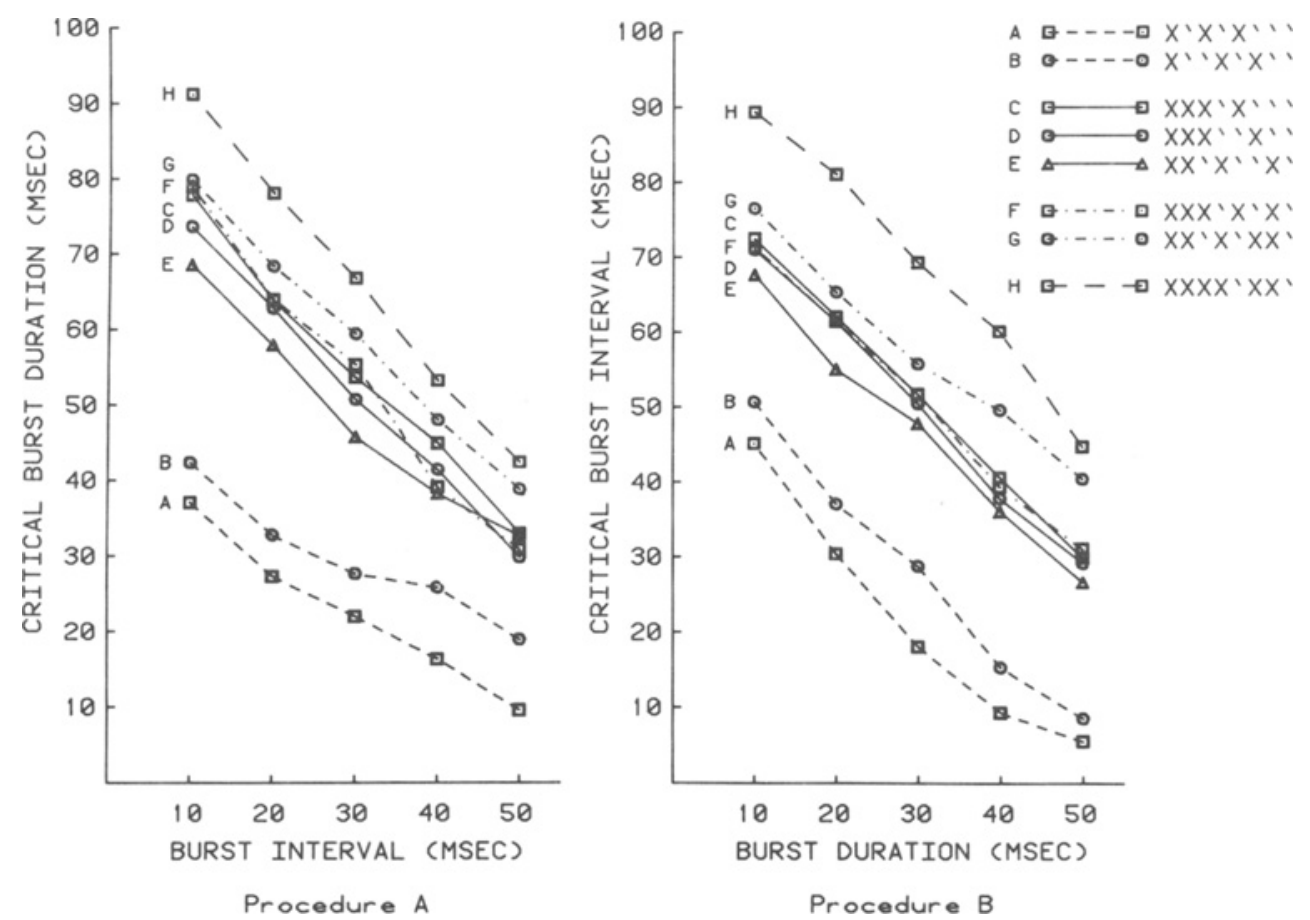

Figure 2. (Left panel) Critical tone-burst duration as a function of interburst interval. (Right panel) Critical interburst interval as a function of tone-burst duration for eight patterns. In the legend, the $X$ represents a tone burst followed by an interburst interval; the symbol' represents a silent period equal to the sum of the tone-burst duration and the interburst interval.

The data for critical durations and intervals for all eight patterns were submitted to analyses of variance. For fixed durations, the mean critical intervals for patterns differed significantly $[F(7,14)=38.21, \mathrm{p}<.0001]$, as did the means for durations $[\mathrm{F}(4,8)=50.48, \mathrm{p}<.0001]$. The interaction was not significant $(F<1.00)$. For fixed intervals, the mean critical durations for patterns differed significantly $[F(7,14)=25.68, p<.0001]$, as did the means for intervals $[F(4,8)=129.98, p<.0001]$. The interaction was significant $[\mathrm{F}(28,56)=3.29, \mathrm{p}<.001]$. The interaction appears to be due to values for Patterns $A$ and $B$ at extreme burst durations.

In choosing patterns, we had selected one relatively ambiguously organizing and one unambiguously organizing pattern with 3,4 , and 5 bursts; the ambiguous patterns were $B, E$, and $G$, and the unambiguous patterns were $A, C$, and F. Patterns $D$ and $H$ were not used in this analysis. A separate three-way analysis of variance was performed using the data for these six patterns in order to examine the effects of the number of bursts, ambiguity, and interval or duration.

The results of the analysis of variance of critical interval $I_{1}$ were the same as for the global analysis of variance involving all eight patterns. Significant effects were found only for the number of bursts $[F(2,4)=43.56, p<$ $.01]$ and for intervals $[F(4,8)=37.47, p<.0001]$. The means for the ambiguous patterns were in a direction consistent with predictions that might be made on the basis of spectral characteristics of unambiguous (metrical or regular) and ambiguous (ametrical or irregular) sequence structures, but the differences were not significant $[\mathrm{F}(2,4)$ $=8.96, \mathrm{p}<.10]$. Results for critical burst duration also mirrored those for critical interval. Significant effects were found for number of bursts $[F(2,4)=37.54, p<$ $.01]$ and for intervals $[F(4,8)=74.63, p<.0001]$; the interaction of number of bursts and intervals was also significant $[F(8,16)=3.80, p=.01]$. The effect of ambiguity was not significant $(F<1.00)$; an interaction of ambiguity with the number of bursts fell just short of significance $[F(2,4)=5.84, p=.06]$. The differences between ambiguous and unambiguous patterns in temporal dispersion or metricality of pattern structure were apparently not large enough to attain significant effects.

A final observation is that the mean durations and intervals for pattern $\mathrm{C}$, a four-burst pattern, are about twice those for pattern A, a three-burst pattern. In general, the mean values for four-burst patterns are about twice those for three-burst patterns. If three-burst Pattern A, $X^{\prime} X^{\prime} X^{\prime \prime \prime}$, is examined in terms of Martin's (1972) theory, it reduces to a four-beat pattern in which sounds are present on the first three beats. When considered in this way, the pattern is one in which the interburst intervals in Procedure $A$ are not $I_{1}$ (i.e, 10, 20,30, 40, and $50 \mathrm{msec}$ ), but are $I_{1}$ plus $I_{2}$ (i.e, $2 I_{1}+D$ ); hence, the pattern length is four, not eight. This is not true for Pattern B, however, because the shortest interval between one burst's 
offset and another's onset is the experimentally manipulated $\mathrm{I}_{1}$.

\section{DISCUSSION}

The results presented demonstrate that the perceived unitization of a cyclically repeating pattern of a sound burst is a reliable, measurable phenomenon. The results satisfy the expectations of a simple lowpass filter model that would have a time constant that smooths the envelope of the pattern elements such that, at some repetition rate, all elements are smoothed into a single cyclic waveform. The relationships between tone-burst duration and interburst interval are linear, except perhaps for critical $I_{1}$ of three-element patterns. If the phenomenon is related to the spectrum of the pattern that is input to a filter, one should find, as we have, that duration and interval for a particular pattern will always be in a relationship such that the sum of the two will be a constant. This is because the sum determines the fundamental frequency of the cyclic pattern and the fundamental frequency at which unitization occurs should remain the same for each experimental variation of duration or interval.

Perhaps the most intriguing findings are that these constants vary as the number of bursts in the patterns vary and that the constant for four-burst patterns is twice that of three-burst patterns. The theoretical stance taken here is that it is a consequence of filtering, hence a consequence of the spectrum of the patterns. Thus, we performed Fourier analyses to determine the spectrum of each pattern.

\section{SPECTRAL ANALYSES}

\section{Method}

According to the model, the modulating signal is a function that varies between zero and unity and when multiplied by the carrier frequency generates the auditory signal presented to the subject's ear. Because the modulating signal creates the temporal pattern, the analysis is confined to this signal. A digital representation of each signal was constructed with a length of 512 samples. Digitization was based on data for the $30-\mathrm{msec}$ interburst interval. For convenience of scaling in subsequent plotting of the magnitude spectra, a signal amplitude of 10 was assumed for the pattern function which was constructed of 10s (burst on) and 0s (burst off) instead of $1 \mathrm{~s}$ and $0 \mathrm{~s}$.

The magnitude and phase spectra of digitized patterns were calculated by the fast Fourier transform (FFT) algorithm of Brigham (1974).

\section{Results}

The magnitudes of the first 128 harmonics of the spectra of the patterns are shown in Figure 3. There are several observations to be made from these spectra.

The first feature of importance is that in both of the three-burst patterns, the greatest amount of energy is concentrated around the fourth harmonic. In Pattern $\mathrm{X}^{\prime} \mathrm{X}^{\prime} \mathrm{X}^{\prime \prime \prime}$, the largest amplitude component is the fourth harmonic, in Pattern $\mathrm{X}^{\prime \prime} \mathrm{X}^{\prime} \mathrm{X}^{\prime \prime}$, the largest amplitude component is the third and considerable energy is present in the fourth, fifth, sixth, and seventh harmonics. However, in the remaining patterns, the largest amplitude component is the eighth harmonic. That is, the patterns with durations and intervals that are approximately twice those
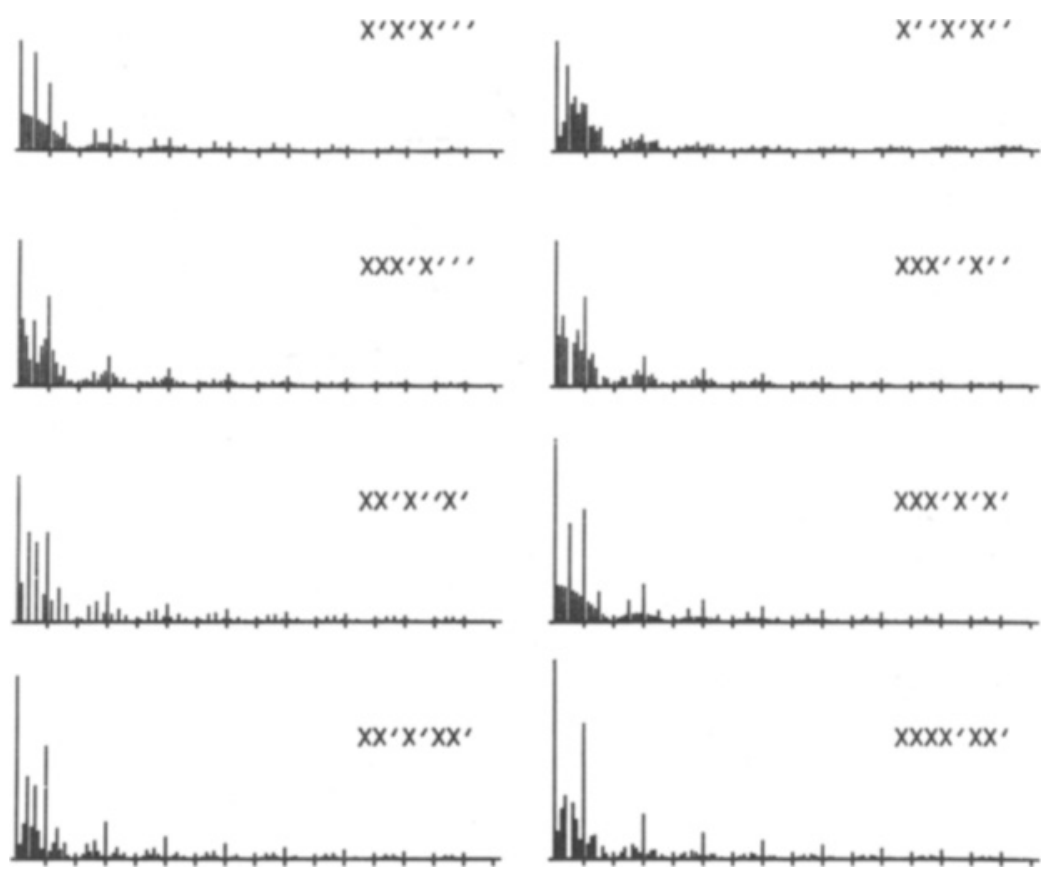

Figure 3. Magnitude spectra for eight unary patterns. The magnitude for the zeroth frequency (so-called "DC level") is the first line. Ticks on the abscissa mark off the eighth harmonic and succeeding harmonics in multiples of eight. The spectra are normalized with respect to their harmonic frequencies. See text for details of the Fourier analysis. 
of the three-burst patterns have spectra in which the largest amplitude harmonic is twice that of the largest amplitude harmonic of the three-burst patterns.

The spectra presented in Figure 3 show the harmonic frequencies for waveforms of standard length (i.e., one pattern cycle sampled at 512 points). To compare the spectra with respect to their temporal frequencies (in hertz or cycles per second), the spectra of several patterns were replotted in Figure 4. From the period of the pattern cycle at which unitization was achieved, the temporal frequency of the fundamental harmonic was determined (i.e., the fundamental frequency in hertz is the reciprocal of the pattern cycle's period in time). The fundamental and higher harmonics were aligned in the figure according to their temporal frequency.

The second feature of importance is the spectral characteristics of patterns that are perceptually unambiguous or ambiguous. The unambiguous patterns, Patterns A, C, and $F$, all have significant energy in the first, second, fourth, and eighth harmonics, which are in octave rela- tionships. The ambiguous patterns-Patterns $X^{\prime \prime} X^{\prime} X^{\prime \prime}$, $X X X^{\prime \prime} X^{\prime \prime}, X X^{\prime} X^{\prime \prime} X^{\prime}$, and $X X^{\prime} X^{\prime} X X^{\prime}$-all have significant energy distributed in the third, fifth, seventh, and ninth harmonics in addition to the second, fourth, and eighth. Thus, perceived regularity or irregularity seems to be associated with the way spectral energy is distributed among harmonics.

\section{GENERAL DISCUSSION}

The results of the experiments and the spectral analyses support the conception of a lowpass filter model of temporal-pattern perceptual experience of unitization occurs for a particular pattern at a particular temporal interval between bursts and some multiple of this interval (eight in these experiments) which is the presentation interval. Comparisons of spectral analyses of the patterns indicated that a shift of the fundamental frequency of the pattern, which occurs when the duration and interburst interval are adjusted to generate the perceptual phenome-

$x^{\prime} x^{\prime \prime \prime} x^{\prime \prime \prime}$
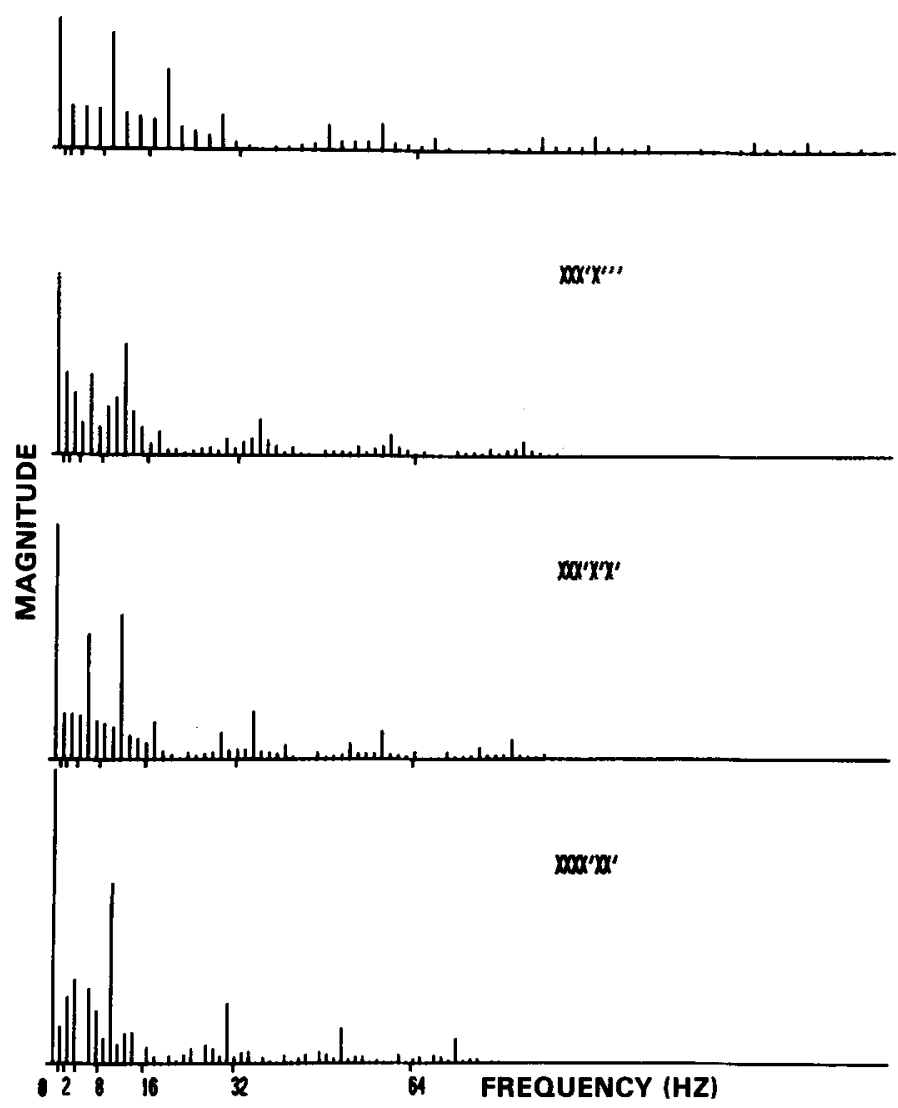

Figure 4. Magnitude spectra for patterns with three, four, five, and six bursts in which the first 64 harmonic frequencies are aligned according to their actual temporal frequency in hertz when unitization was achieved. The first line of the spectrum gives the amplitude of the zeroth frequency (DC). The second line is the fundamental frequency of the pattern based on the period of one pattern cycle when the subjects reported hearing unitization. 
non, aligns the harmonics that have greatest amplitude at approximately the same temporal frequency. This is consistent with what is necessary for the hypothetical filter to produce the same extent of smoothing of the envelope of the signal for all patterns.

The experimental work and the model are relevant to the work on polyrhythms done by Handel and Oshinsky (1981). They found that, when tapping out polyrhythms, subjects responded to the sequence (rhythm) with the larger number of elements at slow presentation rates, shifted to the sequence with the smaller number of elements at fast rates, and tended to give "unit" responses as the repetition rate of the sequences increased. The choice of this response occurred most often between 0.8 and $0.4 \mathrm{sec}$ per pattern repetition. The pattern repetition rates at which perceived unitization occurred in our study was between $0.44 \mathrm{sec}$ per pattern repetition for Pattern $\mathrm{X}^{\prime} \mathrm{X}^{\prime} \mathrm{X}^{\prime \prime \prime}$ and $0.79 \mathrm{sec}$ for Pattern $\mathrm{XXXX}^{\prime} \mathrm{XX}^{\prime}$ at a 30 msec interburst interval in Procedure A. It seems probable that the "unit" motor response was influenced by perceptual unitization. The evidence indicates that both polyrhythmic and "monorhythmic" stimuli are perceptually unitized by a lowpass filter mechanism.

The shifts from one rhythm (i.e., on part of the pattern) to another made by subjects in Handel and Oshinsky (1981) may have occurred because the filter attenuates the frequencies that define one pattern more than it attenuates the frequencies of the other. The frequencies that would be attenuated most will be the higher frequencies, of course. When the pattern contains a train of five pulses distributed over the same time interval as another train of two pulses, the spectral frequencies defining the greater number of pulses must be higher than those defining the smaller number of pulses. We would expect the waveform of the train with more pulses to be affected earlier than that of the train with fewer pulses.

Our work is also relevant to the work of Martin (1972) and Jones (1976). Martin proposed that accents occur at equally spaced points in a rhythmic structure, and that any rhythm could therefore be described hierarchically on the basis of temporal grouping from the smallest interval between notes to the larger repeating interval. We have seen that such regularity is reflected in the temporal frequency spectrum. The observations of Martin may reflect an auditory processing system that searches out the rhythmicity of various sound sources by evaluating the temporal frequency spectrum for a harmonic structure in which significant energy is present in the octave harmonics of the fundamental. The information provided for such processing would be the output of the lowpass filter.

When the results for unambiguous or metrical patterns are compared with those for the ambiguous or ametrical patterns, spectral characteristics emerge as important determinants of temporal pattern perception. To say that ametrical patterns do not have perceived accents falling naturally at equally spaced intervals, as Martin (1972) does, is simply to describe a phenomenon and does not suggest what exists in the physical properties of a stimulus that creates the phenomenon. Our observations about the spectra of the metrical and ametrical patterns indicate that concentration of energy in octave frequencies of the temporal pattern spectrum is associated with easily perceived, strong organization. Others (Bregman, Abramson, Doehring, \& Darwin, 1985; Broadbent \& Ladefoged, 1957; Darwin, 1981; Parsons, 1976) have proposed that the isolation of two or more sound sources is based on the harmonics generated by the sources. In a multiplexed amplitude modulation system, this is easily understood. But it is especially interesting that Handel and Oshinsky (1981) used polyrhythms generated with sound bursts that had identical frequencies. That two trains are perceived and tracked by the subject is an indication that the temporal frequency spectrum contains information defining two different temporal frequency harmonic structures that are differentially affected by filtering.

\section{REFERENCES}

BREGMAN, A. (1981). Asking the "What for" question in auditory perception. In M. Kubovy \& J. R. Pomerantz (Eds.), Perceptual organization. Hillsdale, NJ: Erlbaum

Bregman, A., Abramson, J., Doehring, P., \& Darwin, C. (1985). Spectral integration based on common amplitude modulation. Perception \& Psychophysics, 37, 483-493.

Brigham, E. O. (1974). The fast Fourier transform. Englewood Cliffs, NJ: Prentice-Hall.

Broadbent, D. E., \& LAdefoged, P. (1957). On the fusion of sounds reaching different sense organs. Journal of the Acoustical Society of America, 29, 708-710.

BroadBent, D. E., \& LAdEFOGED, P. (1959). Auditory perception of temporal order. Joumal of the Acoustical Society of America, 31, 1539.

DARWIN, C. J. (1981). Perceptual grouping of speech components differing in fundamental frequency and onset-time. Quarterly Journal of Experimental Psychology, 33A, 185-207.

Fraisse, P. (1982). Rhythm and tempo. In D. Deutch (Ed.), The psychology of music. New York: Academic Press.

GARNER, W. R., \& GoTTwaLd, R. L. (1968). The perception and learning of temporal patterns. Quarterly Journal of Experimental Psychology, 20, 97-109.

GINSBURG, A. P. (1978). Visual information processing based on spatial filters constrained by biological data (Report AMRL-RT-78-129). Aerospace Medical Research Laboratory, Wright-Patterson Air Force Base, Ohio.

HANDEL, S. (1973). Temporal segmentation of repeating auditory patterns. Journal of Experimental Psychology, 101, 46-54.

Handel, S. (1974). Perceiving melodic and rhythmic auditory patterns. Journal of Experimental Psychology, 103, 922-933.

HANDEL, S., \& OSHINSKY, J. S. (1981). The meter of syncopated auditory polyrhythms. Perception \& Psychophysics, 30, 1-9.

JoNES, M. R. (1976). Time, our lost dimension: Toward a new theory of perception, attention, and memory. Psychological Review, 82, 323-355.

JoNES, M. R. (1981). A tutorial on some issues and methods in serial patterns research. Perception \& Psychophysics, 30, 492-504.

Kubovy, M. (1981). Concurrent-pitch segregation and the theory of indispensable attributes. In M. Kubovy \& J. R. Pomerantz (Eds.), Perceptual organization. Hillsdale, NJ: Erlbaum.

MARTIN, J. G. (1972). Rhythmic (hierarchical) versus serial structure in speech and other behavior. Psychological Review, 79, 487-509.

Parsons, T. W. (1976). Separation of speech from interfering speech by means of harmonic selection. Journal of the Acoustical Society of America, 60, $911-918$.

Preusser, D. (1972). The effect of structure and rate on the recogni- 
tion and description of auditory temporal patterns. Perception \& Psychophysics, 11, 233-240.

Preusser, D., Garner, W. R., \& Gottwald, R. L. (1970). Perceptual organization of two-element temporal patterns as a function of their component one-element patterns. American Journal of Psychology, 83, 151-170.

ROYER, F. L., \& GARNER, W. R. (1966). Response uncertainty and perceptual difficulty of auditory temporal patterns. Perception \& Psychophysics, 1, 41-47.

ROYer, F. L., \& GARNER, W. R. (1970). Perceptual organization of nine-element auditory temporal patterns. Perception \& Psychophysics, $7,115-120$.

Sturges, P. S., \& Martin, J. G. (1974). Rhythmic structure in audi- tory temporal pattern perception and immediate memory. Journal of Experimental Psychology, 102, 377-383.

VIEMEISTER, N. F. (1979). Temporal modulation transfer functions based upon modulation thresholds. Journal of the Acoustical Society of America, 66, 1364-1380.

Warren, R. M., Obusek, C. J., Farmer, R. M., \& Warren, R. P. (1969). Auditory sequences: Confusions of patterns other than speech or music. Science, 164, 586-587.

YOST, W. A., \& NIELSEN, D. W. (1977). Fundamentals of hearing: An introduction. New York: Holt, Rinehart, \& Winston.

(Manuscript received May 3, 1985;

revision accepted for publication November 11,1985 .) 